J. Korean Math. Soc. 50 (2013), No. 3, pp. 607-625

http://dx.doi.org/10.4134/JKMS.2013.50.3.607

\title{
REFLECTION PRINCIPLES FOR GENERAL WIENER FUNCTION SPACES
}

\author{
Ian Pierce And David Skoug
}

\begin{abstract}
It is well-known that the ordinary single-parameter Wiener space exhibits a reflection principle. In this paper we establish a reflection principle for a generalized one-parameter Wiener space and apply it to the integration of a class of functionals on this space. We also discuss several notions of a reflection principle for the two-parameter Wiener space, and explore whether these actually hold.
\end{abstract}

\section{Introduction}

Let $C_{0}[0, T]$ denote single-parameter Wiener space; this is the space of $\mathbb{R}$ valued continuous functions on $[0, T]$ with $x(0)=0$. Let $\mathcal{M}$ denote the class of Wiener measurable subsets of $C_{0}[0, T]$ and let $\mathfrak{m}$ denote Wiener measure. Then $\left(C_{0}[0, T], \mathcal{M}, \mathfrak{m}\right)$ is a complete measure space and we denote the integral of a Wiener-measurable functional $F$ by $\int_{C_{0}[0, T]} F(x) \mathfrak{m}(d x)$ whenever this integral exists. Note that the point evaluation functional $\left\langle\delta_{t}, x\right\rangle=x(t)$ is an $\mathbb{R}$-valued random variable with mean

$$
\mathbb{E}[x(t)]=\int_{C_{0}[0, T]} x(t) \mathfrak{m}(d x)=0
$$

and covariance

$$
\mathbb{E}[x(s) x(t)]=\int_{C_{0}[0, T]} x(s) x(t) \mathfrak{m}(d x)=\min (s, t) ;
$$

observe also that $t \mapsto x(t)$ is a realization of the standard Brownian motion process.

It is well-known that the Wiener space $C_{0}[0, T]$ exhibits a reflection principle; that is for all $c \geq 0$,

$$
\mathfrak{m}\left\{x: \sup _{[0, T]} x(t) \geq c\right\}=2 \mathfrak{m}\{x: x(T) \geq c\} .
$$

Received September 26, 2012.

2010 Mathematics Subject Classification. 28C20, 46G12, 60 G20.

Key words and phrases. generalized Wiener space, Yeh-Wiener space, generalized Brownian motion, distribution of supremum. 
Proofs and discussions of this result can be found in $[12,14,23]$ and elsewhere; a particularly good explanation is given in [2].

In Section 2, we show that the generalized function space $C_{a, b}[0, T]$ exhibits a very similar behavior about its mean function $a(t)$; that is, for $c \geq 0$,

$$
\mu\left\{x: \sup _{[0, T]}[x(t)-a(t)] \geq c\right\}=2 \mu\{x:[x(T)-a(T)] \geq c\} .
$$

This fact in turn leads to formulas aiding in the integration of a class of functionals on $C_{a, b}[0, T]$.

For $Q=[0, S] \times[0, T]$, let $C_{2}(Q)$ denote the two parameter Wiener space (see [21]); this is the space of all $\mathbb{R}$-valued continuous functions on $Q$ satisfying $x(s, 0)=x(0, t)=0$ for all $(s, t) \in Q$. In Sections 3 and 4 , we consider the difficulties in formulating a notion similar to that expressed in equations (1) and (2).

\section{Greatest deviations on the function space $C_{a, b}[0, T]$}

We first introduce the generalized Wiener space $\left(C_{a, b}[0, T], \mathcal{M}\left(C_{a, b}[0, T]\right), \mu\right)$. We follow the same formulation as $[5,6,8]$. Let $a$ and $b$ be functions defined on $[0, T]$ with $a^{\prime} \in L^{2}[0, T]$, and $b^{\prime}$ continuous, positive, and bounded away from 0 on $[0, T]$. Observe that $a$ and $b$ are absolutely continuous and $b$ is strictly increasing on $[0, T]$, and so one can define a generalized Brownian motion as in Chapter 3 of [23]. We take $\mu$ to be the Gaussian measure on $C[0, T]$ with finite-dimensional distributions having density

$$
\left(\prod_{j=1}^{n} 2 \pi\left[b\left(t_{j}\right)-b\left(t_{j-1}\right)\right]\right)^{-\frac{1}{2}} \exp \left(-\frac{1}{2} \sum_{j=1}^{n} \frac{\left(\left[u_{j}-a\left(t_{j}\right)\right]-\left[u_{j-1}-a\left(t_{j-1}\right)\right]\right)^{2}}{b\left(t_{j}\right)-b\left(t_{j-1}\right)}\right)
$$

with $t_{0}=0, u_{0}=0$, and $0=a(0)=b(0)$.

Let $\|\cdot\|$ denote the usual supremum norm and let $C_{a, b}[0, T]$ denote the Banach space $\{x \in C[0, T]: x(0)=0\}$ on which this measure $\mu$ is supported (as shown in [23]). Then $\left(C_{a, b}[0, T], \mathcal{B}\left(C_{a, b}[0, T]\right), \mu\right)$ is a measure space, where $\mathcal{B}\left(C_{a, b}[0, T]\right)$ is the Borel sigma algebra of $C_{a, b}[0, T]$. One can then complete this measure space to $\left(C_{a, b}[0, T], \mathcal{M}\left(C_{a, b}[0, T]\right), \mu\right)$, with $\mathcal{M}\left(C_{a, b}[0, T]\right)$ denoting the complete sigma algebra of Wiener measurable subsets.

We note that with respect to $\mu$ the coordinate evaluation map $\left\langle\delta_{t}, x\right\rangle=x(t)$ is the generalized Brownian motion process determined by $a$ and $b$, having mean

$$
\mathbb{E}[x(t)]=\int_{C_{a, b}[0, T]} x(t) \mu(d x)=a(t),
$$

and covariance function

$$
r(s, t)=\int_{C_{a, b}[0, T]}[x(s)-a(s)][x(t)-a(t)] \mu(d x)=\min \{b(s), b(t)\} .
$$


For more information about these function spaces, consult $[5,6,7,8,9,10]$.

We will refer to the map $x \mapsto \sup _{[0, T]}[x(t)-a(t)]$ as the greatest (positive) deviation function on the space $C_{a, b}[0, T]$. In the same way one may take the greatest (negative) deviation function as $x \mapsto \sup _{[0, T]}[a(t)-x(t)]$. As its name suggests, this function measures the largest amount of deviation of an element $x \in C_{a, b}[0, T]$ from the center of the support of $\mu$ (i.e. the function $a$ ). Note also that

$$
\|x-a\|=\max \left\{\sup _{[0, T]}[x(t)-a(t)], \sup _{[0, T]}[a(t)-x(t)]\right\} .
$$

We will make use of the following lemma from Chapter 3 of [23].

Lemma 1. Let $\left\{X_{j}: j=1, \ldots, n\right\}$ be an independent set of symmetrically distributed random variables on a probability space $(\Omega, \mathcal{B}, \mathbb{P})$, let $S_{0}=0$, and let $S_{j}=X_{1}+\cdots+X_{j}$ for $j=1, \ldots, n$. Then for every $\varepsilon>0$,

$$
2 \mathbb{P}\left[S_{n} \geq c\right] \geq \mathbb{P}\left[\max _{1 \leq j \leq n} S_{j} \geq c\right] \geq 2 \mathbb{P}\left[S_{n} \geq c+2 \varepsilon\right]-2 \sum_{j=1}^{n} \mathbb{P}\left[X_{j} \geq \varepsilon\right] .
$$

We are now ready to establish equation (2) above, which will yield valuable information about the behavior of the greatest deviation function. Our proof uses ideas from Chapter 3 of [23] as well as from unpublished lecture notes of R. H. Cameron.

Theorem 1. For all $c \geq 0$,

$$
\mu\left\{x: \sup _{[0, T]}[x(t)-a(t)] \geq c\right\}=2 \mu\{x:[x(T)-a(T)] \geq c\} .
$$

Proof. Let $D \subseteq[0, T]$ be countable and dense, containing 0 and $T$, and let $P_{n}=\left\{0=t_{0}<t_{1}<\cdots<t_{n}=T\right\}$ denote a nested sequence of partitions of $[0, T]$ with each $t_{j} \in D$ and $\operatorname{mesh}\left(P_{n}\right) \rightarrow 0$ as $n \rightarrow \infty$. Note that the process $X_{t}=x(t)$ is continuous and separable, and thus for all $c>0$ and $\varepsilon>0$,

$$
\begin{aligned}
\mu\left\{x: \sup _{[0, T]}[x(t)-a(t)] \geq c\right\} & =\mu\left\{x: \sup _{D}[x(t)-a(t)] \geq c\right\} \\
& \leq \mu\left(\bigcup_{n=1}^{\infty}\left\{x: \max _{1 \leq k \leq n+1}\left[x\left(t_{k}\right)-a\left(t_{k}\right)\right] \geq c-\varepsilon\right\}\right) \\
& =\lim _{n \rightarrow \infty} \mu\left\{x: \max _{1 \leq k \leq n+1}\left[x\left(t_{k}\right)-a\left(t_{k}\right)\right] \geq c-\varepsilon\right\} \\
& \leq 2 \mu\{x: x(T)-a(T) \geq c-\varepsilon\},
\end{aligned}
$$

where the last inequality is due to Lemma 1 . Taking the limit as $\varepsilon \rightarrow 0$ yields

$$
\mu\left\{x: \sup _{[0, T]}[x(t)-a(t)] \geq c\right\} \leq 2 \mu\{x:[x(T)-a(T)] \geq c\} .
$$


For the other inequality, we specify partitions $P_{n}=\left\{0=t_{0}<t_{1}<\cdots<\right.$ $\left.t_{n}=T\right\}$ with $t_{k}=\frac{k T}{n}$. Then for any $c>0$ and $\varepsilon>0$ we again use Lemma 1 to obtain

$$
\begin{aligned}
& \mu\left\{x: \sup _{[0, T]}[x(t)-a(t)] \geq c\right\} \\
\geq & \mu\left\{x: \max _{1 \leq k \leq n}\left[x\left(t_{k}\right)-a\left(t_{k}\right)\right] \geq c\right\} \\
\geq & 2 \mu\{x:[x(T)-a(T)] \geq c+2 \varepsilon\} \\
& -2 \sum_{k=1}^{n} \mu\left\{x:\left[x\left(t_{k}\right)-a\left(t_{k}\right)-x\left(t_{k-1}\right)+a\left(t_{k-1}\right)\right] \geq \varepsilon\right\} .
\end{aligned}
$$

We estimate

$$
\begin{aligned}
\frac{1}{\sqrt{2 \pi s}} \int_{\varepsilon}^{\infty} \exp \left(-\frac{u^{2}}{2 s}\right) \mathrm{d} u & \leq \frac{1}{\sqrt{2 \pi s}} \int_{\varepsilon}^{\infty} \exp \left(-\frac{\varepsilon u}{2 s}\right) \mathrm{d} u \\
& =\frac{\sqrt{2 s}}{\varepsilon \sqrt{\pi}} \exp \left(-\frac{\varepsilon^{2}}{2 s}\right)
\end{aligned}
$$

and (noting that $b^{\prime}$ is positive and bounded) that

$$
\begin{aligned}
\max _{1 \leq k \leq n}\left[b\left(t_{k}\right)-b\left(t_{k-1}\right)\right] & =\max _{1 \leq k \leq n} \int_{t_{k-1}}^{t_{k}} b^{\prime}(s) \mathrm{d} s \\
& \leq \max _{1 \leq k \leq n}\left(\left[t_{k}-t_{k-1}\right] \int_{t_{k-1}}^{t_{k}}\left(b^{\prime}(s)\right)^{2} \mathrm{~d} s\right)^{\frac{1}{2}} \\
& \leq \frac{\left\|b^{\prime}\right\|_{2} \sqrt{T}}{\sqrt{n}} .
\end{aligned}
$$

Recall that $x\left(t_{k}\right)-a\left(t_{k}\right)-x\left(t_{k-1}\right)+a\left(t_{k-1}\right)$ is distributed normally with mean 0 and variance $b\left(t_{k}\right)-b\left(t_{k-1}\right)$, and then using our estimates in (6) and (7), we find that

$$
\begin{aligned}
& \lim _{n \rightarrow \infty} \sum_{k=1}^{n} \mu\left\{x:\left[x\left(t_{k}\right)-a\left(t_{k}\right)-x\left(t_{k-1}\right)+a\left(t_{k-1}\right)\right] \geq \varepsilon\right\} \\
= & \lim _{n \rightarrow \infty} \sum_{k=1}^{n} \frac{1}{\sqrt{2 \pi\left[b\left(t_{k}\right)-b\left(t_{k-1}\right)\right]}} \int_{\varepsilon}^{\infty} \exp \left(-\frac{u^{2}}{b\left(t_{k}\right)-b\left(t_{k-1}\right)}\right) \mathrm{d} u \\
\leq & \lim _{n \rightarrow \infty} \sum_{k=1}^{n} \frac{\sqrt{2\left[b\left(t_{k}\right)-b\left(t_{k-1}\right)\right]}}{\varepsilon \sqrt{\pi}} \exp \left(-\frac{\varepsilon^{2}}{2\left[b\left(t_{k}\right)-b\left(t_{k-1}\right)\right]}\right) \\
\leq & \lim _{n \rightarrow \infty} n\left[\frac{\sqrt{2\left\|b^{\prime}\right\|_{2} \sqrt{T}}}{\varepsilon \sqrt{\pi n}} \exp \left(-\frac{\varepsilon^{2}}{2\left\|b^{\prime}\right\|_{2} \sqrt{T}}\right)\right]
\end{aligned}
$$




$$
\leq \lim _{n \rightarrow \infty} \sum_{k=1}^{n} \frac{\sqrt{2 n\left\|b^{\prime}\right\|_{2} \sqrt{T}}}{\varepsilon \sqrt{\pi}} \exp \left(-\frac{\sqrt{n} \varepsilon^{2}}{2\left\|b^{\prime}\right\|_{2} \sqrt{T}}\right)
$$

Now use this estimate and let $n \rightarrow \infty$ and then $\varepsilon \rightarrow 0$ in (5).

The theorem above has several useful corollaries.

Corollary 1. Let $E$ be a Lebesgue measurable subset of $\mathbb{R}$. Then

$$
\mu\left\{x: \sup _{[0, T]}[x(t)-a(t)] \in E\right\}=2 \mu\{x:[x(T)-a(T)] \in E \cap[0, \infty)\}
$$

and

$$
\begin{aligned}
& \int_{C_{a, b}[0, T]} \chi_{E}\left(\sup _{[0, T]}[x(t)-a(t)]\right) \mu(d x) \\
= & \frac{2}{\sqrt{2 \pi b(T)}} \int_{0}^{\infty} \chi_{E}(u) \exp \left(-\frac{u^{2}}{2 b(T)}\right) \mathrm{d} u .
\end{aligned}
$$

Proof. The proof is a standard exercise in measure theory. Begin with $E$ an open interval and the result follows easily. The case for an open set $E$ follows by decomposing $E$ into a countable union of disjoint intervals. From this, demonstrate that (8) holds for $G_{\delta}$ and then null sets. Finally use this to demonstrate the conclusion for Lebesgue measurable sets. Then (9) follows immediately from (8).

In the next corollary we note that by choosing the mean function $a$ to be identically zero, we immediately recover a direct extension of the reflection principle for ordinary Wiener space, as expected.

Corollary 2. Let $\mu$ be a generalized Wiener measure on $C_{a, b}[0, T]$ with $a(t)=0$ on $[0, T]$. Then for all $c \geq 0$ and $t_{0} \in(0, T]$,

$$
\mu\left\{x: \sup _{\left[0, t_{0}\right]} x(t) \geq c\right\}=2 \mu\left\{x: x\left(t_{0}\right) \geq c\right\} .
$$

Corollary 3. Let $f: \mathbb{R} \rightarrow \mathbb{C}$ be Lebesgue measurable with $f=0$ on $(-\infty, 0)$ and put $F(x)=f\left(\sup _{[0, T]}[x(t)-a(t)]\right)$. Then $F$ is $\mu$-measurable and

$$
\begin{aligned}
\int_{C_{a, b}[0, T]} F(x) \mu(d x) & =\int_{C_{a, b}[0, T]} f\left(\sup _{[0, T]}[x(t)-a(t)]\right) \mu(d x) \\
& =2 \int_{C_{a, b}[0, T]} f(x(T)-a(T)) \mu(d x) \\
& =\frac{2}{\sqrt{2 \pi b(T)}} \int_{0}^{\infty} f(u) \exp \left(-\frac{u^{2}}{2 b(T)}\right) \mathrm{d} u .
\end{aligned}
$$


Let $p$ be a positive real number; for $c \geq 0$ we let

$$
\operatorname{erfc}(c ; p)=\frac{1}{\sqrt{2 \pi p}} \int_{c}^{\infty} \exp \left(-\frac{u^{2}}{2 p}\right) \mathrm{d} u
$$

denote the complimentary error function with variance parameter $p$. Also let $X(x)=\sup _{[0, T]}[x(t)-a(t)]$ be the greatest deviation as a random variable on the probability space $\left(C_{a, b}[0, T], \mathcal{M}, \mu\right)$. As an application of Corollaries 1 and 3 we obtain the following information about this random variable.

Corollary 4. Let $X(x)=\sup _{[0, T]}[x(t)-a(t)]$. Then

(1) the cumulative distribution function for $X$ is

$$
F(c)= \begin{cases}0 & \text { if } c<0 \\ 1-2 \operatorname{erfc}(c ; b(T)) & \text { if } c \geq 0\end{cases}
$$

(2) the probability density function for $X$ is

$$
f(c)= \begin{cases}0 & \text { if } c<0 \\ \frac{2}{\sqrt{2 \pi b(T)}} \exp \left(-\frac{c^{2}}{2 b(T)}\right) & \text { if } c \geq 0\end{cases}
$$

(3) the first moment (expectation) of $X$ is $\mathbb{E}[X]=\sqrt{\frac{2 b(T)}{\pi}}$,

(4) the variance of $X$ is $\operatorname{Var}[X]=b(T)-\frac{2 b(T)}{\pi}$,

(5) the characteristic function for $X$ is analytic, with series representation

$$
\varphi(\xi)=\sum_{k=0}^{\infty} a_{k}(i \xi)^{k},
$$

where the coefficients $a_{k}$ are of the form

$$
a_{k}=\frac{(2 b(T))^{\frac{k}{2}}}{k ! \sqrt{\pi}} \Gamma\left(\frac{k+1}{2}\right) .
$$

Proof. It is an immediate consequence of Corollary 1 that

$$
\mu\left\{x: \sup _{[0, T]}[x(t)-a(t)] \geq c\right\}=2 \operatorname{erfc}(c, b(T)) .
$$

Observe that the cdf for $X$ is

$$
F(c)=\mathbb{P}[X \leq c]=1-\mu\left\{x: \sup _{[0, T]}[x(t)-a(t)] \geq c\right\},
$$

and then note that the right hand side of this equation is 0 if $c<0$ and is equal to $1-2 \operatorname{erfc}(c, b(T))$ otherwise. Differentiating $F$ yields $f$.

Using Corollary 3, straightforward computations show that

$$
\mathbb{E}[X]=\frac{2}{\sqrt{2 \pi b(T)}} \int_{0}^{\infty} u \exp \left(-\frac{u^{2}}{2 b(T)}\right) \mathrm{d} u=\sqrt{\frac{2 b(T)}{\pi}},
$$


and also that

$$
\begin{aligned}
\mathbb{E}\left[X^{2}\right] & =\frac{2}{\sqrt{2 \pi b(T)}} \int_{0}^{\infty} u^{2} \exp \left(-\frac{u^{2}}{2 b(T)}\right) \mathrm{d} u \\
& =\frac{2}{\sqrt{2 \pi b(T)}}\left(\sqrt{2}(b(T))^{\frac{3}{2}} \int_{0}^{\infty} v^{\frac{1}{2}} \exp (-v) \mathrm{d} v\right) \\
& =\frac{2 b(T)}{\sqrt{\pi}} \Gamma\left(\frac{3}{2}\right) \\
& =b(T) .
\end{aligned}
$$

Then the variance of $X$ is easily determined.

We obtain the characteristic function for $X$ by calculating

$$
\begin{aligned}
\varphi(\xi) & =\int_{C_{a, b}[0, T]} \exp \left(i \xi \sup _{[0, T]}[x(t)-a(t)]\right) \mu(d x) \\
& =\frac{2}{\sqrt{2 \pi b(T)}} \int_{0}^{\infty} \exp (i u \xi) \exp \left(-\frac{u^{2}}{2 b(T)}\right) \mathrm{d} u .
\end{aligned}
$$

For $z \in \mathbb{C}$ we consider the function

$$
F(z)=\int_{0}^{\infty} \frac{2}{\sqrt{2 \pi b(T)}} \exp (i u z) \exp \left(-\frac{u^{2}}{2 b(T)}\right) \mathrm{d} u .
$$

Note that the integrand

$$
f(z, u)=\frac{2}{\sqrt{2 \pi b(T)}} \exp (i u z) \exp \left(-\frac{u^{2}}{2 b(T)}\right)
$$

is measurable on $[0, \infty)$ for each $z \in \mathbb{C}$ and is entire for each $u \in[0, \infty)$. In addition, for any $z_{0} \in \mathbb{C}$ and $\delta>0$ the function

$$
\sup _{\left|z-z_{0}\right|<\delta}|f(z, \cdot)|
$$

is integrable on $[0, \infty)$ because

$$
\begin{aligned}
\left|f(z, u)-f\left(z_{0}, u\right)\right| & =\frac{2}{\sqrt{2 \pi b(T)}} \exp \left(-\frac{u^{2}}{2 b(T)}\right)\left|e^{i u z}-e^{i u z_{0}}\right| \\
& \leq \frac{2}{\sqrt{2 \pi b(T)}} \exp \left(-\frac{u^{2}}{2 b(T)}\right)\left(2 \exp \left(u \operatorname{Im} z_{0}+\delta\right)\right)
\end{aligned}
$$

whenever $\left|z-z_{0}\right|<\delta$. Then by [15] the function $F$ is entire, with

$$
\frac{d}{d z} F(z)=\int_{0}^{\infty} \frac{\partial}{\partial z} f(z, u) \mathrm{d} u
$$

From this we see that

$$
\frac{d^{k}}{d z^{k}} F(z)=\frac{2}{\sqrt{2 \pi b(T)}} \int_{0}^{\infty}(i u)^{k} \exp (i u z) \exp \left(-\frac{u^{2}}{2 b(T)}\right) \mathrm{d} u,
$$


and then expanding $F$ as a power series centered at $z=0$ we obtain the desired coefficients by computing

$$
\begin{aligned}
F^{(k)}(0) & =\frac{2 i^{k}}{\sqrt{2 \pi b(T)}} \int_{0}^{\infty} u^{k} \exp \left(-\frac{u^{2}}{2 b(T)}\right) \mathrm{d} u \\
& =\frac{i^{k}}{\sqrt{2 \pi b(T)}}(2 b(T))^{\frac{k+1}{2}} \int_{0}^{\infty} v^{\frac{k-1}{2}} e^{-v} \mathrm{~d} v \\
& =\frac{i^{k}(2 b(T))^{\frac{k}{2}}}{\sqrt{\pi}} \Gamma\left(\frac{k+1}{2}\right) .
\end{aligned}
$$

Restricting $F$ to $\mathbb{R}$, we see that $\varphi(\xi)$ has the desired properties.

We have some additional corollaries, which can be used to yield error estimates when approximating function space integrals using interpolation by tame functionals. For examples of the use of these types of results, see [3, 20].

Corollary 5. If $f$ is Lebesgue measurable and nonnegative on $[0, \infty)$, then $f(\|x-a\|)$ is $\mu$-measurable and

$$
\int_{C_{a, b}[0, T]} f(\|x-a\|) \mu(d x) \leq \frac{4}{\sqrt{2 \pi b(T)}} \int_{0}^{\infty} f(u) \exp \left(-\frac{u^{2}}{2 b(T)}\right) \mathrm{d} u .
$$

Proof. Partition $C_{a, b}[0, T]$ into

$$
A=\left\{x: \sup _{[0, T]}[x(t)-a(t)] \geq \sup _{[0, T]}[a(t)-x(t)]\right\}
$$

and

$$
B=\left\{x: \sup _{[0, T]}[a(t)-x(t)]>\sup _{[0, T]}[x(t)-a(t)]\right\} .
$$

Then we find that

$$
\begin{aligned}
\int_{C_{a, b}[0, T]} f(\|x-a\|) \mu(d x)= & \int_{A} f(\|x-a\|) \mu(d x)+\int_{B} f(\|x-a\|) \mu(d x) \\
= & \int_{A} f\left(\sup _{[0, T]}[x(t)-a(t)]\right) \mu(d x) \\
& +\int_{B} f\left(\sup _{[0, T]}[a(t)-x(t)]\right) \mu(d x) \\
\leq & \int_{C_{a, b}[0, T]} f\left(\sup _{[0, T]}[x(t)-a(t)]\right) \mu(d x) \\
& \quad+\int_{C_{a, b}[0, T]} f\left(\begin{array}{l}
\left.\sup _{[0, T]}[a(t)-x(t)]\right) \mu(d x)
\end{array}\right.
\end{aligned}
$$




$$
=\frac{4}{\sqrt{2 \pi b(T)}} \int_{0}^{\infty} f(u) \exp \left(-\frac{u^{2}}{2 b(T)}\right) \mathrm{d} u
$$

where the last equality follows from Corollary 3 , the positivity of $f$, and the symmetry of the centered normal distribution.

Corollary 6. Let $f$ be Lebesgue measurable and monotonically increasing on $[0, \infty)$. Then

$$
\frac{2}{\sqrt{2 \pi b(T)}} \int_{0}^{\infty} f(u) \exp \left(-\frac{u^{2}}{2 b(T)}\right) \mathrm{d} u \leq \int_{C_{a, b}[0, T]} f(\|x-a\|) \mu(d x)
$$

whenever both sides are defined. Moreover, if $f$ is Lebesgue measurable and monotonically decreasing the reverse inequality holds whenever both sides are defined.

Proof. Recall that

$$
\|x-a\|=\max \left\{\sup _{[0, T]}[x(t)-a(t)], \sup _{[0, T]}[a(t)-x(t)]\right\},
$$

Using this fact, the monotonicity of $f$, and Corollary 3 , we have

$$
\begin{aligned}
\frac{2}{\sqrt{2 \pi b(T)}} \int_{0}^{\infty} f(u) \exp \left(-\frac{u^{2}}{2 b(T)}\right) \mathrm{d} u & =\int_{C_{a, b}[0, T]} f\left(\sup _{[0, T]}[x(t)-a(t)]\right) \mu(d x) \\
& \leq \int_{C_{a, b}[0, T]} f(\|x-a\|) \mu(d x)
\end{aligned}
$$

as desired. For decreasing $f$ the inequality clearly reverses.

A final corollary follows immediately from the previous two.

Corollary 7. If $f$ is Lebesgue measurable, nonnegative, and monotonically increasing on $[0, \infty)$, then there exists some $M$ satisfying $2 \leq M \leq 4$ such that

$$
\int_{C_{a, b}[0, T]} f(\|x-a\|) \mu(d x)=\frac{M}{\sqrt{2 \pi b(T)}} \int_{0}^{\infty} f(u) \exp \left(-\frac{u^{2}}{2 b(T)}\right) \mathrm{d} u
$$

\section{No reflection principle for two-parameter Wiener space}

Let $Q=[0, S] \times[0, T]$ and $\partial Q=\{(s, t) \in Q: s=0, S$ or $t=0, T\}$ be the boundary of $Q$, and let $C_{2}(Q)$ denote the space of continuous $\mathbb{R}$-valued functions defined on $Q$ for which $x(0, t)=x(s, 0)=0$. In [21, 22], Yeh constructed a Gaussian measure $\mathfrak{m}_{y}$ on $C_{2}(Q)$ with respect to which the point evaluation functional $\delta_{(s, t)}$ is an $\mathbb{R}$-valued random variable having mean

$$
\mathbb{E}[x(s, t)]=\int_{C_{2}(Q)} x(s, t) \mathfrak{m}_{y}(d x)=0
$$


and covariance

$$
\mathbb{E}[x(s, t) x(u, v)]=\int_{C_{2}(Q)} x(s, t) x(u, v) \mathfrak{m}_{y}(d x)=\min (s, u) \min (t, v) .
$$

Recall that for functions of one variable, the classical notion of a function of bounded variation is unambiguously defined and very well understood. However, when considering functions of two (or more) variables, there are many possible definitions for the concept of bounded variation. See $[1,11,19]$ for several such definitions and a considerable amount of discussion.

In the same way, for multiple parameter Wiener spaces, one can formulate the idea of a reflection principle in a variety of manners. In this section, we will consider several such formulations and determine whether the space $C_{2}(Q)$ satisfies each of them.

For ordinary single parameter Wiener space, we note that $x(0)=0$ for $x \in$ $C_{0}[0, T]$; therefore considering again the single parameter reflection principle, we see that

$$
\begin{aligned}
\mathfrak{m}\left\{x: \sup _{[0, T]} x(t) \geq c\right\}= & 2 \mathfrak{m}\{x: x(T) \geq c\} \\
= & 2 \mathfrak{m}\{x: x(T) \geq c ; x(0) \geq c\} \\
& +2 \mathfrak{m}\{x: x(T) \geq c ; x(0)<c\} \\
= & 2 \mathfrak{m}\left\{x: \max _{\{0, T\}} x(t) \geq c\right\}
\end{aligned}
$$

for $c \geq 0$. From this, we might consider the reflection principle to be a means of expressing either of the following:

(1) a relationship between the behavior of the supremum $x(t)$ on the interval to its behavior at the endpoint $T$ of the interval, or

(2) a relationship between the behavior of the supremum $x(t)$ on the interval to its behavior on the boundary $\{0, T\}$ of the interval.

We immediately have two candidate formulations for a reflection principle in the two parameter setting; we can ask the following corresponding questions:

(1) Is there a constant $k_{1} \geq 0$ so that for every $c \geq 0$,

$$
\mathfrak{m}_{y}\left\{x: \sup _{Q} x(s, t) \geq c\right\}=k_{1} \mathfrak{m}_{y}\{x: x(S, T) \geq c\} ?
$$

(2) Is there a constant $k_{2} \geq 0$ so that for every $c \geq 0$,

$$
\mathfrak{m}_{y}\left\{x: \sup _{Q} x(s, t) \geq c\right\}=k_{2} \mathfrak{m}_{y}\left\{x: \sup _{\partial Q} x(s, t) \geq c\right\} ?
$$

In fact, the answer to both questions is negative, as we will demonstrate below. 
We wish to compare $\mathfrak{m}_{y}\left\{x: \sup _{Q} x(s, t) \geq c\right\}$ with either $\mathfrak{m}_{y}\{x: x(S, T) \geq c\}$ or $\mathfrak{m}_{y}\left\{x: \sup _{\partial Q} x(s, t) \geq c\right\}$; therefore we define

$$
\gamma_{1}(c)=\frac{\mathfrak{m}_{y}\left\{x: \sup _{Q} x(s, t) \geq c\right\}}{\mathfrak{m}_{y}\{x: x(S, T) \geq c\}}
$$

and

$$
\gamma_{2}(c)=\frac{\mathfrak{m}_{y}\left\{x: \sup _{Q} x(s, t) \geq c\right\}}{\mathfrak{m}_{y}\left\{x: \sup _{\partial Q} x(s, t) \geq c\right\}} .
$$

Observe that both $\gamma_{1}$ and $\gamma_{2}$ are continuous on $[0, \infty)$; moreover it is easy to see that $\gamma_{1}(0)=2$ and $\gamma_{2}(0)=1$. Also, in [24], Zimmerman shows that $\gamma_{1}(c) \leq 4$ for all $c \geq 0$.

\subsection{Negative answer to the first question}

For each $c \geq 0$, we consider the following sets:

$$
\begin{aligned}
& A_{c}=\left\{x: \sup _{[0, S]} x(s, T) \geq c\right\}, \\
& B_{c}=\left\{x: \sup _{Q} x(s, t) \geq c ; \sup _{[0, S]} x(s, T)<c\right\},
\end{aligned}
$$

and

$$
D_{c}=\left\{x: \sup _{Q} x(s, t)<c\right\} .
$$

It is clear that $A_{c}, B_{c}$, and $D_{c}$ are disjoint and that

$$
C_{2}(Q)=A_{c} \cup B_{c} \cup D_{c},
$$

and putting $f(c)=\mathfrak{m}_{y}\left(A_{c}\right), g(c)=\mathfrak{m}_{y}\left(B_{c}\right)$, and $h(c)=\mathfrak{m}_{y}\left(D_{c}\right)$, we observe that

$$
1=f(c)+g(c)+h(c) .
$$

We will make use of the following theorem of Cameron and Storvick from [4].

Theorem 2. Let $F$ be a functional defined on $C_{0}[0, S]$ such that $F(\sqrt{T} w)$ is a Wiener measurable functional of $w$ on $C_{0}[0, S]$. Then $F(x(\cdot, T))$ is a YehWiener measurable functional of $x$ on $C_{2}(Q)$ and

$$
\int_{C_{2}(Q)} F(x(\cdot, T)) \mathfrak{m}_{y}(d x)=\int_{C_{0}[0, S]} F(\sqrt{T} w) \mathfrak{m}(d w),
$$

where the existence of either integral implies the existence of the other with equality. 
Remark 1. As shown by Skoug in [18], the hypothesis of measurability in the previous theorem can be assumed either for $F(x)$ on $C_{2}(Q)$ or for $F(\sqrt{T} w)$ on $C_{0}[0, S]$, and the measurability of one will imply the measurability of the other.

Using this theorem, we demonstrate that, as one would reasonably expect, the space $C_{2}(Q)$ exhibits a reflection principle when restricted to any horizontal or vertical line in $Q$.

Proposition 1. For $c \geq 0$,

$$
f(c)=\mathfrak{m}_{y}\left\{x: \sup _{[0, S]} x(s, T) \geq c\right\}=2 \mathfrak{m}_{y}\{x: x(S, T) \geq c\}=2 \operatorname{erfc}(c, S T) .
$$

Proof. Using (1) and (24) above, a computation and an easy change of variable show that

$$
\begin{aligned}
f(c) & =\int_{C_{2}(Q)} \chi_{[c, \infty)}\left(\sup _{[0, S]} x(s, T)\right) \mathfrak{m}_{y}(d x) \\
& =\int_{C_{0}[0, S]} \chi_{[c, \infty)}\left(\sup _{[0, S]} \sqrt{T} w(s)\right) \mathfrak{m}(d w) \\
& =\frac{2}{\sqrt{2 \pi S}} \int_{0}^{\infty} \chi_{[c, \infty)}(\sqrt{T} u) \exp \left(-\frac{u^{2}}{2 S}\right) \mathrm{d} u \\
& =\frac{2}{\sqrt{2 \pi S T}} \int_{0}^{\infty} \chi_{[c, \infty)}(u) \exp \left(-\frac{u^{2}}{2 S T}\right) \mathrm{d} u \\
& =\frac{2}{\sqrt{2 \pi S T}} \int_{c}^{\infty} \exp \left(-\frac{u^{2}}{2 S T}\right) \mathrm{d} u \\
& =2 \mathfrak{m}_{y}\{x: x(S, T) \geq c\}
\end{aligned}
$$

for each $c \geq 0$.

The next lemma follows readily from equations (21), (22), (23), and (25) above.

\section{Lemma 2.}

(1) The function $f(c)$ is smooth, strictly decreasing, and concave upward on $[0, \infty)$. Furthermore, $f(0)=1, \lim _{c \rightarrow \infty} f(c)=0$, and $f$ has a fixed point in $(0,1)$.

(2) The function $h(c)=\mathfrak{m}_{y}\left(D_{c}\right)$ is continuous and strictly increasing on $[0, \infty)$, with $h(0)=0, \lim _{c \rightarrow \infty} h(c)=1$, and $h(c)=1-c$ for some $c$ in $(0,1)$.

(3) The function $g(c)=\mathfrak{m}_{y}\left(B_{c}\right)$ is continuous on $[0, \infty)$, with $g(0)=0$, $\lim _{c \rightarrow \infty} g(c)=0$ and $f(c)+g(c)=c$ for some $c$ in $(0,1)$.

Now, using (17), (21), (23), and (25), it follows that

$$
\gamma_{1}(c)=\frac{2 \mathfrak{m}_{y}\left\{x: \sup _{Q} x(s, t) \geq c\right\}}{\mathfrak{m}_{y}\left(A_{c}\right)}
$$




$$
\begin{aligned}
& =\frac{2(1-h(c))}{f(c)} \\
& =\frac{2(f(c)+g(c))}{f(c)} \\
& =2+\frac{2 g(c)}{f(c)} \\
& \geq 2
\end{aligned}
$$

for each $c \geq 0$. Moreover, $2=\gamma_{1}(0) \leq \gamma_{1}(c) \leq 4$ by Zimmerman's result; this and (26) imply that

$$
0=g(0) \leq g(c) \leq f(c) \leq f(0)=1
$$

on $[0, \infty)$. Now, $2=\gamma_{1}(0)=2+\frac{2 g(c)}{f(c)}$, so that if $\gamma_{1}$ is to be equal to a constant $k_{1}$ it must be the case that $g$ is identically zero on $[0, \infty)$.

We now show that this cannot be true. For $c>0$, put

$$
\phi(s, t)= \begin{cases}\frac{8 c}{S T} & \text { if } 0 \leq s \leq \frac{S}{2}, 0 \leq t \leq \frac{T}{2} \\ -\frac{8 c}{S T} & \text { if } \frac{S}{2}<s \leq S, 0 \leq t \leq \frac{T}{2} \\ -\frac{8 c}{S T} & \text { if } \frac{T}{2}<t \leq T, 0 \leq s \leq \frac{S}{2} \\ \frac{8 c}{S T} & \text { if } \frac{S}{2}<s \leq S, \frac{T}{2}<t \leq T\end{cases}
$$

and let

$$
x_{0}(s, t)=\int_{0}^{s} \int_{0}^{t} \phi(u, v) \mathrm{d} v \mathrm{~d} u
$$

Note that $x_{0}\left(\frac{S}{2}, \frac{T}{2}\right)=2 c$ and $x_{0}=0$ on $\partial Q$. Denote by $\mathrm{B}\left(x_{0} ; \frac{c}{2}\right)$ the ball of radius $\frac{c}{2}$ around $x_{0}$ and observe that this ball is contained in the set $B_{c}$, whence

$$
\mathfrak{m}_{y}\left(\mathrm{~B}\left(x_{0} ; \frac{c}{2}\right)\right) \leq \mathfrak{m}_{y}\left(B_{c}\right)=g(c) .
$$

As $\phi$ is of bounded variation in the sense of Hardy-Krause (see [1] for explanation), we apply the Cameron-Martin theorem for $C_{2}(Q)$ as found in [22] to see that

$$
\begin{aligned}
\mathfrak{m}_{y}\left(\mathrm{~B}\left(x_{0} ; \frac{c}{2}\right)\right) & =\int_{\mathrm{B}\left(x_{0}, \frac{c}{2}\right)} \mathrm{m}_{y}(d x) \\
& =\int_{\mathrm{B}\left(0, \frac{c}{2}\right)} \exp \left(-\frac{1}{2}\|\phi\|_{2}^{2}+\int_{Q} \phi(s, t) \mathrm{d} x(s, t)\right) \mathrm{m}_{y}(d x) \\
& \geq \exp \left(-\frac{32 c^{2}}{S T}\right) \int_{\mathrm{B}\left(0, \frac{c}{2}\right)} \exp \left(-\frac{36 c^{2}}{S T}\right) \mathrm{m}_{y}(d x) \\
& \geq \exp \left(-\frac{68 c^{2}}{S T}\right) \mathrm{m}_{y}\left(\mathrm{~B}\left(0, \frac{c}{2}\right)\right) \\
& >0
\end{aligned}
$$


where we have used the fact that the stochastic integral $\int_{Q} \phi(s, t) \mathrm{d} x(s, t)$ is equal $\mathfrak{m}_{y}$-a.e. to the ordinary Riemann-Stieltjes integral, whence we can integrate to obtain

$$
\int_{Q} \phi(s, t) \mathrm{d} x(s, t)=\frac{8 c}{S T}\left[x(S, T)+4 x\left(\frac{S}{2}, \frac{T}{2}\right)-2 x\left(S, \frac{T}{2}\right)-2 x\left(\frac{S}{2}, T\right)\right],
$$

which we can easily bound from below on $\mathrm{B}\left(0, \frac{c}{2}\right)$. Thus by $(29)$ we see that $g(c)>0$ whenever $c>0$, and thus (15) cannot hold for any constant $k_{1}$.

\subsection{Negative answer to the second question}

In a similar fashion as above, put

$$
\begin{aligned}
& A_{c}^{\prime}=\left\{x: \sup _{\partial Q} x(s, t) \geq c\right\}, \\
& B_{c}^{\prime}=\left\{x: \sup _{Q} x(s, t) \geq c ; \sup _{\partial Q} x(s, t)<c\right\}
\end{aligned}
$$

and

$$
D_{c}^{\prime}=\left\{x: \sup _{Q} x(s, t)<c\right\} .
$$

As above, $C_{2}(Q)$ is the disjoint union of these sets. We let $F(c)=\mathfrak{m}_{y}\left(A_{c}^{\prime}\right)$, $G(c)=\mathfrak{m}_{y}\left(B_{c}^{\prime}\right)$, and $H(c)=\mathfrak{m}_{y}\left(D_{c}^{\prime}\right)$, so that

$$
1=F(c)+G(c)+H(c) .
$$

From this, we can write

$(35)$

$$
\begin{aligned}
& \gamma_{2}(c) \\
= & \frac{\mathfrak{m}_{y}\left\{x: \sup _{Q} x(s, t) \geq c\right\}}{\mathfrak{m}_{y}\left\{x: \sup _{\partial Q} x(s, t) \geq c\right\}} \\
= & \frac{\mathfrak{m}_{y}\left\{x: \sup _{\partial Q} x(s, t) \geq c\right\}+\mathfrak{m}_{y}\left\{x: \sup _{Q} x(s, t) \geq c ; \sup _{\partial Q} x(s, t)<c\right\}}{\mathfrak{m}_{y}\left\{x: \sup _{\partial Q} x(s, t) \geq c\right\}} \\
= & 1+\frac{G(c)}{F(c)} .
\end{aligned}
$$

As above, the fact that $\gamma_{2}(0)=1$ implies that (16) holds for a constant $k_{2}=1$ only if $G(c)=0$ for all $c$. Taking the same $x_{0}$ as defined in (28), we observe that the ball $\mathrm{B}\left(x_{0} ; \frac{c}{2}\right)$ is contained in $B_{c}^{\prime}$ and then using (30) we can demonstrate that

$$
0<\mathfrak{m}_{y}\left(\mathrm{~B}\left(x_{0} ; \frac{c}{2}\right)\right) \leq \mathfrak{m}_{y}\left(B_{c}^{\prime}\right)=G(c)
$$

for $c>0$, and so (16) cannot hold for any constant $k_{2}$. 
Easily extending results from [16], in [17] we obtain the explicit formula

$$
\begin{aligned}
F(c) & =\mathfrak{m}_{y}\left\{x: \sup _{\partial Q} x(s, t) \geq c\right\} \\
& =\frac{3}{\sqrt{2 \pi S T}} \int_{c}^{\infty} \exp \left(-\frac{u^{2}}{2 S T}\right) \mathrm{d} u-\frac{\exp \left(\frac{4 c^{2}}{S T}\right)}{\sqrt{2 \pi S T}} \int_{3 c}^{\infty} \exp \left(-\frac{u^{2}}{2 S T}\right) \mathrm{d} u \\
& =3 \operatorname{erfc}(c, S T)-\exp \left(\frac{4 c^{2}}{S T}\right) \operatorname{erfc}(3 c, S T) .
\end{aligned}
$$

The following properties of $F, G$, and $H$ follow easily from equations (34), (35), and (36).

\section{Lemma 3.}

(1) The function $F(c)=\mathfrak{m}_{y}\left(A_{c}^{\prime}\right)$ is smooth and strictly decreasing, with $F(0)=1$ and $\lim _{c \rightarrow \infty} F(c)=0$, and $F$ has a fixed point in $(0,1)$.

(2) The function $H(c)=\mathfrak{m}_{y}\left(D_{c}^{\prime}\right)$ is continuous and strictly increasing on $[0, \infty)$, with $H(0)=0, \lim _{c \rightarrow \infty} H(c)=1$, and $H(c)=1-c$ for some $c$ in $(0,1)$.

(3) The function $G(c)=\mathfrak{m}_{y}\left(B_{c}^{\prime}\right)$ is continuous on $[0, \infty)$ with $G(0)=0$, $\lim _{c \rightarrow \infty} G(c)=0$, and $F(c)+G(c)=c$ for some $c$ in $(0,1)$.

\subsection{Other observations and conclusions}

While we are unable to obtain $\gamma_{1}$ and $\gamma_{2}$ explicitly, we collect some observations about their behavior in this section.

Lemma 4. $\lim _{c \rightarrow \infty} \gamma_{1}(c)=4$ and $\gamma_{1}$ has a fixed point in the interval $(2,4)$.

Proof. In [13], for the special case $S=T=1$, Goodman showed (see [17] for our setting) that

$$
\lim _{c \rightarrow \infty} \frac{\mathfrak{m}_{y}\left\{x: \sup _{Q} x(s, t) \geq c\right\}}{\frac{4}{\sqrt{2 \pi S T}} \int_{c}^{\infty} \exp \left(-\frac{u^{2}}{2 S T}\right) \mathrm{d} u}=1 .
$$

Also, by (23) and (26) we see that

$$
\frac{\mathfrak{m}_{y}\left\{x: \sup _{Q} x(s, t) \geq c\right\}}{\frac{4}{\sqrt{2 \pi S T}} \int_{c}^{\infty} \exp \left(-\frac{u^{2}}{2 S T}\right) \mathrm{d} u}=\frac{1-h(c)}{2 f(c)}=\frac{f(c)+g(c)}{2 f(c)}
$$

From (37) and (38) we determine that $\lim _{c \rightarrow \infty} \frac{g(c)}{f(c)}=1$, and then using (26) it is easy to see that

$$
\lim _{c \rightarrow \infty} \gamma_{1}(c)=\lim _{c \rightarrow \infty}\left(2+\frac{2 g(c)}{f(c)}\right)=4 .
$$

The existence of the fixed point now follows immediately from the continuity of $\gamma_{1}$ and the fact that $2<\gamma_{1}(2)<\gamma_{1}(4)<4$. 
Recall that $\gamma_{1}(0)=2$ and $\gamma_{2}(0)=1$, whence

$$
\mathfrak{m}_{y}\{x: x(S, T) \geq c\}=\frac{1}{\sqrt{2 \pi S T}} \int_{c}^{\infty} \exp \left(-\frac{u^{2}}{2 S T}\right) \mathrm{d} u=\operatorname{erfc}(c, S T),
$$

and that $\mathfrak{m}_{y}\left\{x: \sup _{Q} x(s, t) \geq c\right\}=\mathfrak{m}_{y}\left\{x: \sup _{\partial Q} x(s, t) \geq c\right\}=1$ for $c<0$. From this and the results in [17], our final corollary follows immediately.

Corollary 8. The functions $\gamma_{1}$ and $\gamma_{2}$ enjoy the following properties.

(1) $\lim _{c \rightarrow \infty} \gamma_{2}(c)=\frac{3}{2}$ and $\gamma_{2}$ has a fixed point in the interval $\left(1, \frac{3}{2}\right)$.

(2) for $-\infty<c \leq 0, \gamma_{2}(c)=1$ and $\gamma_{1}(c)=(\operatorname{erfc}(c, S T))^{-1}$.

We remark that if any of the functions $\gamma_{1}, \gamma_{2}$, or $G$ could be obtained explicitly then each of the others would be known as well; in addition an explicit expression for the distribution of $\sup _{Q} x(s, t)$ would thus be obtained.

\section{A positive reflection result for $C_{2}(Q)$}

In light of (25), we see a way in which to formulate a reflection principle which will hold for $C_{2}(Q)$. We have a partial result in Proposition 1, but we can quickly extend this in a very natural manner.

Let $\leq$ be a partial order on $Q$ such that $\left(s_{1}, t_{1}\right) \leq\left(s_{2}, t_{2}\right)$ if and only if $s_{1} \leq s_{2}$ and $t_{1} \leq t_{2}$. We will say that a differentiable function $\phi:[0, S] \rightarrow Q$ is a smooth increasing path in $Q$ if it satisfies $\phi\left(s_{1}\right) \leq \phi\left(s_{2}\right)$ whenever $s_{1} \leq s_{2}$, and $0<\left\|\phi^{\prime}(s)\right\|<M$ for some positive $M$ (here $\phi^{\prime}$ is the derivative vector for $\phi$ and $\|\cdot\|$ is the Euclidean norm on $Q$ ).

We begin with a very basic lemma.

Lemma 5. Let $0 \leq s_{1} \leq s_{2} \leq s_{3} \leq s_{4} \leq S$ and $0 \leq t_{1} \leq t_{2} \leq t_{3} \leq$ $t_{4} \leq T$ with each $\left(s_{i}, t_{i}\right)$ distinct for $i=1,2,3,4$. Then the random variables $X=x\left(s_{4}, t_{4}\right)-x\left(s_{3}, t_{3}\right)$ and $X^{\prime}=x\left(s_{2}, t_{2}\right)-x\left(s_{1}, t_{1}\right)$ are independent and symmetrically distributed.

Proof. The proof of independence is essentially a calculation, as

$$
\begin{aligned}
\mathbb{E}\left[X X^{\prime}\right]= & \int_{C_{2}(Q)}\left[x\left(s_{4}, t_{4}\right)-x\left(s_{3}, t_{3}\right)\right]\left[x\left(s_{2}, t_{2}\right)-x\left(s_{1}, t_{1}\right)\right] \mathfrak{m}_{y}(d x) \\
= & \min \left(s_{4}, s_{2}\right) \min \left(t_{4}, t_{2}\right)-\min \left(s_{4}, s_{1}\right) \min \left(t_{4}, t_{1}\right) \\
& \quad-\min \left(s_{3}, s_{2}\right) \min \left(t_{3}, t_{2}\right)+\min \left(s_{3}, s_{1}\right) \min \left(t_{3}, t_{1}\right) \\
= & s_{2} t_{2}-s_{1} t_{1}-s_{2} t_{2}+s_{1} t_{1} \\
= & 0 .
\end{aligned}
$$

Now, the fact that $X$ and $X^{\prime}$ are Gaussian (being the sum of Gaussian random variables), independence and symmetry follow immediately.

Using this and Lemma 1 we can prove the following theorem in essentially the same manner as Theorem 1 . It establishes a reflection principle on $C_{2}(Q)$ 
when our attention is restricted to the behavior of the space only over an increasing path $\phi$ in $Q$.

Theorem 3. Let $\phi:[0, S] \rightarrow Q$ be a smooth increasing path in $Q$ with $\phi(0)=$ $\left(0, t_{0}\right)$ or $\phi(0)=\left(s_{0}, 0\right)$ and let $c \geq 0$. Then

$$
\mathfrak{m}_{y}\left\{x: \sup _{[0, S]} x(\phi(s)) \geq c\right\}=2 \mathfrak{m}_{y}\{x: x(\phi(S)) \geq c\} .
$$

Proof. Note that the condition on $\phi(0)$ guarantees that $x(\phi(0))=0$ and the fact that $0<\left\|\phi^{\prime}\right\|$ both prevents the potential pathologies of a constant path, and combined with the increasing property of $\phi$ ensures that for any $s_{1}<s_{2}<$ $s_{3}<s_{4}$, the points $\left\{\phi\left(s_{i}\right)\right\}$ will satisfy the hypotheses of Lemma 5 .

Now we can use the independence and symmetry guaranteed by Lemma 5 to assure that for $X_{0}=0$ and $X_{k}=x\left(s_{k}\right)-x\left(s_{k-1}\right)$ satisfy the hypotheses of Lemma 1. Then we essentially mimic the proof of Theorem 1, taking $a$ to be the zero function and taking $b(s)=\phi_{1}(s) \phi_{2}(s)$, where $\phi_{1}$ and $\phi_{2}$ are the coordinate functions of $\phi$.

The only point of concern might be the estimate in (7). However, note that $b^{\prime}(s)=\phi_{1}(s) \phi_{2}^{\prime}(s)+\phi_{2}(s) \phi_{1}^{\prime}(s)$ and the condition that $\left\|\phi^{\prime}\right\| \leq M$ is certainly sufficient to bound $\left\|b^{\prime}\right\|_{2}$, so this poses no difficulties.

Note that the theorem certainly holds for any vertical or horizontal path in $Q$, as Proposition 1 would indicate. The restrictions on the path $\phi$ above are fairly strong and can certainly be relaxed, as the following corollary shows.

Corollary 9. Let $\phi:[0, S] \rightarrow Q$ be any continuous function with $\phi(0)=\left(0, t_{0}\right)$ or $\phi(0)=\left(s_{0}, 0\right)$ and let $c \geq 0$. Then

$$
\mathfrak{m}_{y}\left\{x: \sup _{[0, S]} x(\phi(s)) \geq c\right\}=2 \mathfrak{m}_{y}\{x: x(\phi(S)) \geq c\} .
$$

Proof. Observe that there is a sequence of increasing paths $\left\{\phi_{n}\right\} \subseteq C^{1}([0, S], Q)$ converging uniformly to $\phi$. Now, note that

$$
\lim _{n \rightarrow \infty} \chi_{\left\{x: \sup _{[0, S]} x\left(\phi_{n}(s)\right) \geq c\right\}}(x)=\chi_{\left\{x: \sup _{[0, S]} x(\phi(s)) \geq c\right\}}(x)
$$

and also that

$$
\lim _{n \rightarrow \infty} \chi_{\left\{x: x\left(\phi_{n}(S)\right) \geq c\right\}}(x)=\chi_{\{x: x(\phi(S)) \geq c\}}(x)
$$

pointwise in $x$. From this we conclude that

$$
\begin{aligned}
& \mathfrak{m}_{y}\left\{x: \sup _{[0, S]} x(\phi(s)) \geq c\right\}=\lim _{n \rightarrow \infty} \mathfrak{m}_{y}\left\{x: \sup _{[0, S]} x\left(\phi_{n}(s)\right) \geq c\right\} \\
& =\lim _{n \rightarrow \infty} 2 \mathfrak{m}_{y}\left\{x: x\left(\phi_{n}(S)\right) \geq c\right\} \\
& =2 \mathfrak{m}_{y}\{x: x(\phi(S)) \geq c\} \text {, }
\end{aligned}
$$

by dominated covergence. 
We conclude by remarking that the condition $\phi(0)=\left(s_{0}, 0\right)$ or $\phi(0)=\left(0, t_{0}\right)$ can also be relaxed by taking $b(s)=\phi_{1}(s) \phi_{2}(s)-\phi_{1}(0) \phi_{2}(0)$ in the proof of Theorem 4; this results in no difference to the proof or results of the theorem or its corollary.

\section{References}

[1] E. Berkson and T. A. Gillespie, Absolutely continuous functions of two variables and well-bounded operators, J. London Math. Soc. (2) 30 (1984), no. 2, 305-321.

[2] P. Billingsley, Convergence of probability measures, Wiley Series in Probability and Statistics: Probability and Statistics. John Wiley \& Sons Inc., New York, 1999.

[3] R. H. Cameron, A Simpson rule for the numerical evaluation of Wiener's integrals in function space, Duke Math. J. 18 (1951), 111-130.

[4] Robert H. Cameron and David A. Storvick, Two related integrals over spaces of continuous functions, Pacific J. Math. 55 (1974), 19-37.

[5] S. J. Chang, J. G. Choi, and D. Skoug, Integration by parts formulas involving generalized Fourier-Feynman transforms on function space, Trans. Amer. Math. Soc. 355 (2003), no. 7, 2925-2948.

[6] S. J. Chang and D. M. Chung, Conditional function space integrals with applications, Rocky Mountain J. Math. 26 (1996), no. 1, 37-62.

[7] S. J. Chang and H. S. Chung, Generalized Fourier-Wiener function space transforms, J. Korean Math. Soc. 46 (2009), no. 2, 327-345.

[8] S. J. Chang, H. S. Chung, and D. Skoug, Integral transforms of functionals in $L^{2}\left(C_{a, b}[0, T]\right)$, J. Fourier Anal. Appl. 15 (2009), no. 4, 441-462.

[9] - Convolution products, integral transforms and inverse integral transforms of functionals in $L^{2}\left(C_{0}[0, T]\right)$, Integral Transforms Spec. Funct. 21 (2010), no. 1-2, 143151.

[10] S. J. Chang and D. Skoug, Generalized Fourier-Feynman transforms and a first variation on function space, Integral Transforms Spec. Funct. 14 (2003), no. 5, 375-393.

[11] J. A. Clarkson and R. C. Adams, On definitions of bounded variation for functions of two variables, Trans. Amer. Math. Soc. 35 (1933), no. 4, 824-854.

[12] D. Freedman, Brownian Motion and Diffusion, Holden-Day, San Francisco, 1971.

[13] V. Goodman, Distribution estimates for functionals of the two-parameter Wiener process, Ann. Probability 4 (1976), no. 6, 977-982.

[14] I. Karatzas and S. E. Shreve, Brownian motion and stochastic calculus, Graduate Texts in Mathematics 113, Springer-Verlag, New York, 1991.

[15] L. Mattner, Complex differentiation under the integral, Nieuw Arch. Wiskd. (5) 2 (2001), no. $1,32-35$.

[16] S. R. Paranjape and C. Park, Distribution of the supremum of the two-parameter YehWiener process on the boundary, J. Appl. Probability 10 (1973), 875-880.

[17] I. Pierce and D. Skoug, Comparing the distribution of various suprema on two-parameter Wiener space. To appear in Proc. Amer. Math. Soc

[18] D. Skoug, Converses to measurability theorems for Yeh-Wiener space, Proc. Amer. Math. Soc. 57 (1976), no. 2, 304-310.

[19] _ Feynman integrals involving quadratic potentials, stochastic integration formulas, and bounded variation for functions of several variables, Rend. Circ. Mat. Palermo (2) Suppl. No. 17 (1987), 331-347.

[20] J. Yeh, Approximate evaluation of a class of Wiener integrals, Proc. Amer. Math. Soc. 23 (1969), 513-517.

[21] — Wiener measure in a space of functions of two variables, Trans. Amer. Math. Soc. 95 (1960), 433-450. 
[22] — Cameron-Martin translation theorems in the Wiener space of functions of two variables, Trans. Amer. Math. Soc. 107 (1963), 409-420.

[23] _ Stochastic Processes and the Wiener Integral, Pure and Applied Mathematics, 13, Marcel Dekker Inc., New York, 1973.

[24] G. J. Zimmerman, Some sample function properties of the two-parameter Gaussian process, Ann. Math. Statist. 43 (1972), 1235-1246.

IAN PIERCE

Department of Mathematics

Statistics, and Computer Science

St. Olaf College

NoRTHFIELD, MN 55057, USA

E-mail address: pierce@stolaf.edu

DAVID SKOUG

Department of MATHEMATiCS

UNIVERSITY OF NEBRASKA-LINCOLN

LinCOLN, NE 68588, USA

E-mail address: dskoug1@math.unl.edu 